\title{
Synthesis of $\mathrm{GdFeO}_{3}$ nanoparticles via low-temperature reverse co-precipitation: the effect of strong agglomeration on the magnetic behavior
}

\author{
Y. Albadi ${ }^{1,2}$, K. D. Martinson ${ }^{3}$, A. V. Shvidchenko ${ }^{3}$, I. V. Buryanenko ${ }^{4}$, V. G. Semenov ${ }^{5}$, V. I. Popkov ${ }^{3}$ \\ ${ }^{1}$ Saint Petersburg State Institute of Technology, St. Petersburg, 190013, Russia \\ ${ }^{2}$ Al-Baath University, Homs, 77, Syria \\ ${ }^{3}$ Ioffe Institute, St. Petersburg, 194021, Russia \\ ${ }^{4}$ Peter the Great St. Petersburg Polytechnic University, St. Petersburg, 195251, Russia \\ ${ }^{5}$ Saint Petersburg State University, Petergof, St. Petersburg, 198504, Russia \\ albadi.yamen@gmail.com
}

\begin{abstract}
Gadolinium orthoferrite $\left(\mathrm{GdFeO}_{3}\right)$ seems to have potential as a dual-modal contrast agent for magnetic resonance imaging (MRI), thus its preparation in the form of ultrafine superparamagnetic nanoparticles is currently of great interest. In this work, nanocrystalline $\mathrm{GdFeO}_{3}$ was successfully synthesized by the heat treatment $\left(750{ }^{\circ} \mathrm{C}, 4 \mathrm{~h}\right)$ of gadolinium and iron(III) hydroxides reversely co-precipitated at low temperature $\left(0{ }^{\circ} \mathrm{C}\right)$. Initial and resulting powders were analyzed by EDX, SEM, PXRD, Mössbauer spectroscopy, vibration magnetometry, etc. Gadolinium orthoferrite was formed as isometric nanocrystals with an average size of $23 \pm 3 \mathrm{~nm}$, which were strongly agglomerated into clusters of about $200 \mathrm{~nm}$ in diameter. It was shown that the individual $\mathrm{GdFeO}_{3}$ nanocrystals are superparamagnetic, but in the cluster form, they exhibit a collective weak ferromagnetic behavior. After ultrasonic-assisted disintegration of $\mathrm{GdFeO}_{3}$ to a colloidal solution form, these clusters remained stable due to their strong agglomeration and low zeta potential value of $1 \mathrm{mV}$. Thus, it is concluded that the further use of the synthesized $\mathrm{GdFeO}_{3}$ nanoparticles as a basis of MRI contrast agents will be possible only after the suppression of their clustering.
\end{abstract}

Keywords: gadolinium orthoferrite; nanoparticles; colloidal solutions; magnetic resonance imaging; contrast agents.

Received: 3 April 2020

\section{Introduction}

The action of contrast agents for magnetic resonance imaging (MRI) is based on the reduction of the longitudinal $\left(T_{1}\right)$ and transverse $\left(T_{2}\right)$ relaxation times of water protons [1]. Shortening the longitudinal relaxation time causes increased signal intensity on $T_{1}$-weighted images and the affected regions appear brighter (positive contrast agents), whereas shortening the transverse relaxation time causes decreased signal intensity on $T_{2}$-weighted images and the affected regions appear darker (negative contrast agents) [2]. Almost all MRI contrast agents affect both $T_{1}$ and $T_{2}$ relaxation times, but their effect is usually more pronounced for either the positive or the negative mode of MRI diagnostics; such contrast agents are called single-modal [3]. However, due to the peculiarities of their composition and structure, some substances are capable of exhibiting a hybrid contrast effect and can affect both positive and negative MRI images [4]. Dual-modal (hybrid) contrast agents combine the advantages of single-modal contrast agents and increase diagnostic accuracy, which is carried out in $T_{1}$ and $T_{2}$ modes within the same procedure of an MRI investigation [5]. Therefore, the development of a new generation of MRI contrast agents has scientific and practical importance.

One of these possible substances is gadolinium orthoferrite $\left(\mathrm{GdFeO}_{3}\right)$, which has been previously suggested as an MRI contrast agent in the form of nanoparticles [6-9]. The perovskite structure of $\mathrm{GdFeO}_{3}$ contains gadolinium, which is involved in $T_{1}$-contrast agents $[10,11]$, and iron oxide, which is used as a $T_{2}$-contrast agent as nanoparticles $[12,13]$. Therefore, nanocrystalline gadolinium orthoferrite seems to be promising as a dual-modal $T_{1}-T_{2}$ contrast agent for MRI. A variety of methods have been used in the synthesis of $\mathrm{GdFeO}_{3}$ nanoparticles, including co-precipitation [14], hydrothermal [15], combustion [16], sol-gel [17], microwave [18], and other methods. Among them, the co-precipitation method is a priority for medical applications since it allows the production of gadolinium orthoferrite nanoparticles with minimal chemical impurities. But the traditional direct co-precipitation technique, routinely realized at room temperature, does not allow to obtain superparamagnetic particles of small $(<30 \mathrm{~nm})$ size [14]. Thus, new original approaches are required to solve the synthesis problem of gadolinium orthoferrite nanocrystals with the required crystalline, morphological, and magnetic parameters.

In this paper, the low-temperature reverse co-precipitation technique was proposed to synthesize ultrafine superparamagnetic nanoparticles of $\mathrm{GdFeO}_{3}$. A detailed investigation has been performed to determine their structural and morphological features, as well as magnetic behavior. After that, a colloidal solution based on the as-synthesized 
$\mathrm{GdFeO}_{3}$ nanoparticles was prepared to estimate its compliance with the basic requirements for the solutions of MRI contrast agents.

\section{Experimental}

\subsection{Synthesis of $\mathrm{GdFeO}_{3}$ nanoparticles}

An aqueous solution of stoichiometric amounts of gadolinium nitrate hexahydrate $\mathrm{Gd}\left(\mathrm{NO}_{3}\right)_{3} \cdot 6 \mathrm{H}_{2} \mathrm{O}$ (puriss., 99.9\%) and iron(III) nitrate nonahydrate $\mathrm{Fe}\left(\mathrm{NO}_{3}\right)_{3} \cdot 9 \mathrm{H}_{2} \mathrm{O}$ (pur., $98.0 \%$ ), with a concentration of $0.01 \mathrm{~mol} / \mathrm{L}$ for each salt, was used as a source of gadolinium ions $\mathrm{Gd}^{3+}$ and iron(III) ions $\mathrm{Fe}^{3+}$. An aqueous solution of ammonia with a concentration of $1 \mathrm{~mol} / \mathrm{L}$ prepared from aqueous ammonia $\mathrm{NH}_{4} \mathrm{OH}$ (puriss. spec., 23.5\%) was used as the precipitating medium. The co-precipitation was carried out at a temperature close to $0{ }^{\circ} \mathrm{C}$ by adding the solution of nitrates in a drop-wise manner to the solution of ammonia which was on a magnetic stirrer. The obtained precipitate was washed several times with distilled water. Then the sample was dried at a temperature of $45^{\circ} \mathrm{C}$ for 24 hours. After that, the dried co-precipitated hydroxides were heated at a temperature of $750{ }^{\circ} \mathrm{C}$ for 4 hours in the air.

\subsection{Characterization of the co-precipitated hydroxides and the heat-treated product}

A small amount of the sample of co-precipitated hydroxides was investigated by thermogravimetric analysis (TGA) using a Shimadzu DTG-60 simultaneous thermal analyzer. The content of gadolinium and iron in the coprecipitated hydroxides was determined by energy-dispersive X-ray spectroscopy (EDX) using a TESCAN VEGA3 scanning electron microscope coupled with an X-act EDX microprobe analyzer from Oxford Instruments. The scanning electron microscopy (SEM) images of the co-precipitated hydroxides and the heat-treated product were obtained using the same microscope. The powder X-ray diffraction (PXRD) patterns of the co-precipitated hydroxides and the heat-treated product were obtained using a Rigaku SmartLab 3 diffractometer. The processing of PXRD data was carried out using SmartLab Studio II software. The Brunauer-Emmett-Teller (BET) surface area was determined by nitrogen adsorption-desorption isotherm measurements at $77 \mathrm{~K}$ on a Micromeritics ASAP 2020 nitrogen adsorption apparatus. Pore size distributions of the sample were calculated by the Barrett-Joyner-Halenda (BJH) procedure using adsorption-desorption isotherms. The characteristic particle size of $\mathrm{GdFeO}_{3}$ was estimated by spherical morphology approximation using the formula $D=6 /\left(S \cdot \rho_{X R D}\right)$, where $D$ - characteristic particle size, $S$ - specific surface area, and $\rho_{X R D}-\mathrm{X}$-ray density of $\mathrm{GdFeO}_{3}$.

The state of Fe atoms in the heat-treated product was studied using a WissEl Mössbauer spectrometer. Measurements were made in the absorption geometry at room temperature $(298 \mathrm{~K})$. The isomer shift was evaluated with respect to $\alpha$-Fe. The magnetic properties of the heat-treated product were measured on a Lake Shore 7400 vibrating magnetometer. The magnetic characteristics according to the hysteresis loop data were calculated using the vibration method built in the magnetometer's software on a Lake Shore 7410 vibration magnetometer.

\subsection{Preparation and characterization of the colloidal solution of $\mathrm{GdFeO}_{3}$ nanoparticles}

A colloidal solution of the as-synthesized $\mathrm{GdFeO}_{3}$ nanoparticles was prepared by mixing $10 \mathrm{mg}$ of the heat-treated product with $50 \mathrm{~mL}$ of distilled water and sonicating the mixture for 4 hours. After that, the colloidal solution was centrifugated at a speed of $1000 \mathrm{rpm}$ for 30 minutes, and the supernatant was collected [7]. The size distribution of particles in the prepared colloidal solution was investigated by dynamic light scattering (DLS) using a ZetaSizer Nano ZS analyzer. The zeta potential of particles in the prepared colloidal solution was determined using the same analyzer.

\section{Results and discussion}

Gadolinium and iron(III) hydroxides undergo a series of physicochemical processes before forming gadolinium orthoferrite and its formation begins immediately after the completion of weight loss [14], therefore the TGA of the co-precipitated hydroxides was necessary to determine the minimum temperature at which $\mathrm{GdFeO}_{3}$ forms. The TGA and derivative thermogravimetric (DTG) curves are presented in Fig. 1, and the main weight loss effects are indicated by numbers $1-5$ on the DTG curve.

The total weight loss was about $23.55 \%$. These weight loss effects may include the evaporation of the physically adsorbed water on the sample surface (effect 1), the dehydration of iron(III) hydroxide (effect 2), the dehydration of gadolinium hydroxide (effect 3), the release of the physically adsorbed carbon dioxide $\left(\mathrm{CO}_{2}\right)$ on the sample surface (effect 4), and the decomposition of gadolinium carbonate derivatives with the release of $\mathrm{CO}_{2}$ (effect 5). The decomposition mechanism of gadolinium and iron(III) hydroxides was previously considered in detail in [15] and it was shown to be ending completely at a temperature of $447{ }^{\circ} \mathrm{C}$ which comparable with $\sim 500{ }^{\circ} \mathrm{C}$ in this work. The appearance of carbonate derivatives in the sample of co-precipitated hydroxides may be due to the active sorption of $\mathrm{CO}_{2}$ from the surrounding air on their surface while preparing the solutions of reactants, washing and drying the 


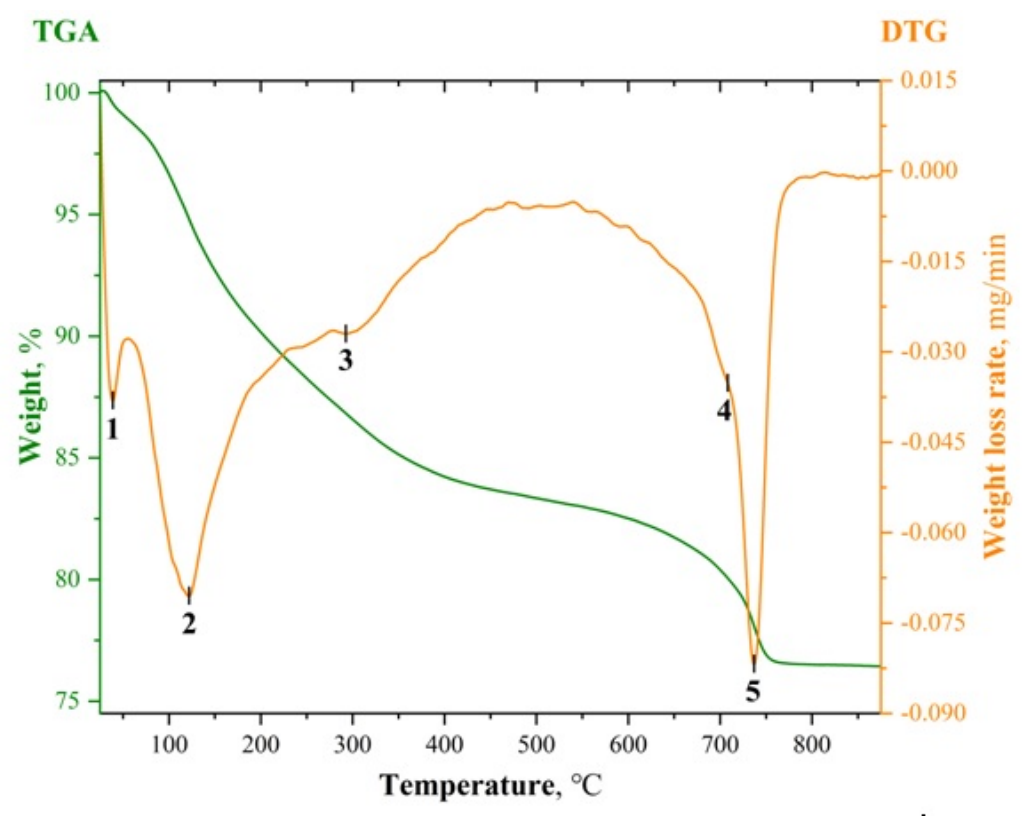

FIG. 1. The TGA and DTG curves of the obtained co-precipitated hydroxides

precipitate. Since the formation of $\mathrm{GdFeO}_{3}$ occurs only after the complete decomposition of gadolinium carbonate derivatives as it was shown in [19] (effect 5, maximum of about $737^{\circ} \mathrm{C}$, a temperature of $750^{\circ} \mathrm{C}$ was chosen as the minimum temperature for the synthesis of $\mathrm{GdFeO}_{3}$ nanoparticles.

The results of EDX, PXRD, and SEM are presented in Fig. 2. The elemental analysis of the obtained sample of the co-precipitated hydroxides by EDX showed that the average stoichiometric gadolinium-to-iron ratio in the obtained sample was $50.31 \%: 49.69 \%$ or 1.00:0.99 which is very close to the ratio of 1:1 as in $\mathrm{GdFeO}_{3}$. The detailed EDX results of co-precipitated hydroxides in terms of gadolinium and iron are shown in the table inserted in Fig. 2a.

According to the diffraction pattern of the co-precipitated hydroxides, they were X-ray amorphous (Fig. 2a). The $\mathrm{X}$-ray phase analysis of the heat-treated product showed that it is mainly composed of orthorhombic gadolinium orthoferrite $\left(o-\mathrm{GdFeO}_{3}\right)$ (Fig. 2b) with a trace amount of a garnet gadolinium ferrite phase called cubic pentairon(III) trigadolinium oxide $\left(c-\mathrm{Gd}_{3} \mathrm{Fe}_{5} \mathrm{O}_{12}\right)$, which is thermodynamically more stable than $\mathrm{GdFeO}_{3}$ [20]. The appearance of this impurity in the sample may be due to the partial carbonization of hydroxides while preparing, which leads to the sample inhomogeneity and, consequently, the formation of another phase along with the main phase after heat treatment. The important role of partial carbonation of the hydroxide precursor on the formation of rare-earth orthoferrites was also previously shown in [21] and [22]. The average crystallite size of the obtained $\mathrm{GdFeO}_{3}$ nanocrystals was calculated by the X-ray line broadening technique based on Scherrer's formula and it is approximately equal to $23 \pm 3 \mathrm{~nm}$. The lognormal size distribution of the prepared $\mathrm{GdFeO}_{3}$ nanocrystals based on the broadening of the reflections (111) and (112) is presented as an insert in Fig. 2b. As for (111), the size distribution is sufficiently narrow, i.e. there is no big difference in crystallite sizes, but in the case of (112), a widening towards larger sizes is observed. This can be explained by the fact that unlike (111), (112) overlaps with other reflections in its region, which complicates the calculation of size distribution. Thus, these results indicate that ultrafine gadolinium orthoferrite nanocrystals with a narrow size distribution have been successfully obtained.

The nanoparticles of the co-precipitated hydroxides were relatively isometric as seen on their SEM image (Fig. 2a). After the heat treatment, this isometric morphology remained the same with a marked decrease in the particle size as compared with the co-precipitated hydroxides (Fig. 2b). This decrease in particle size can be explained by the loss of water and $\mathrm{CO}_{2}$ during the heat treatment (see the TGA results discussed above). The SEM image of the heat-treated product (Fig. 2b) also confirms its narrow crystallite size distribution, i.e. no extra-large particles are observed. These results are in good agreement with the results of the X-ray line broadening analysis given above.

To evaluate the specific surface area of the obtained $\mathrm{GdFeO}_{3}$ nanoparticles, as well as the size and volume of pores in the sample, the low-temperature nitrogen adsorption-desorption measurements were undertaken (Fig. 3).

According to the IUPAC classification, the obtained adsorption isotherm of the heat-treated product belongs to type IV, and the hysteresis loop belongs to type $\mathrm{H} 3$, which suggests that a wide distribution of pore sizes in the sample and that slit-shaped pores are the predominant pore types. These facts can be simply explained by the presence 


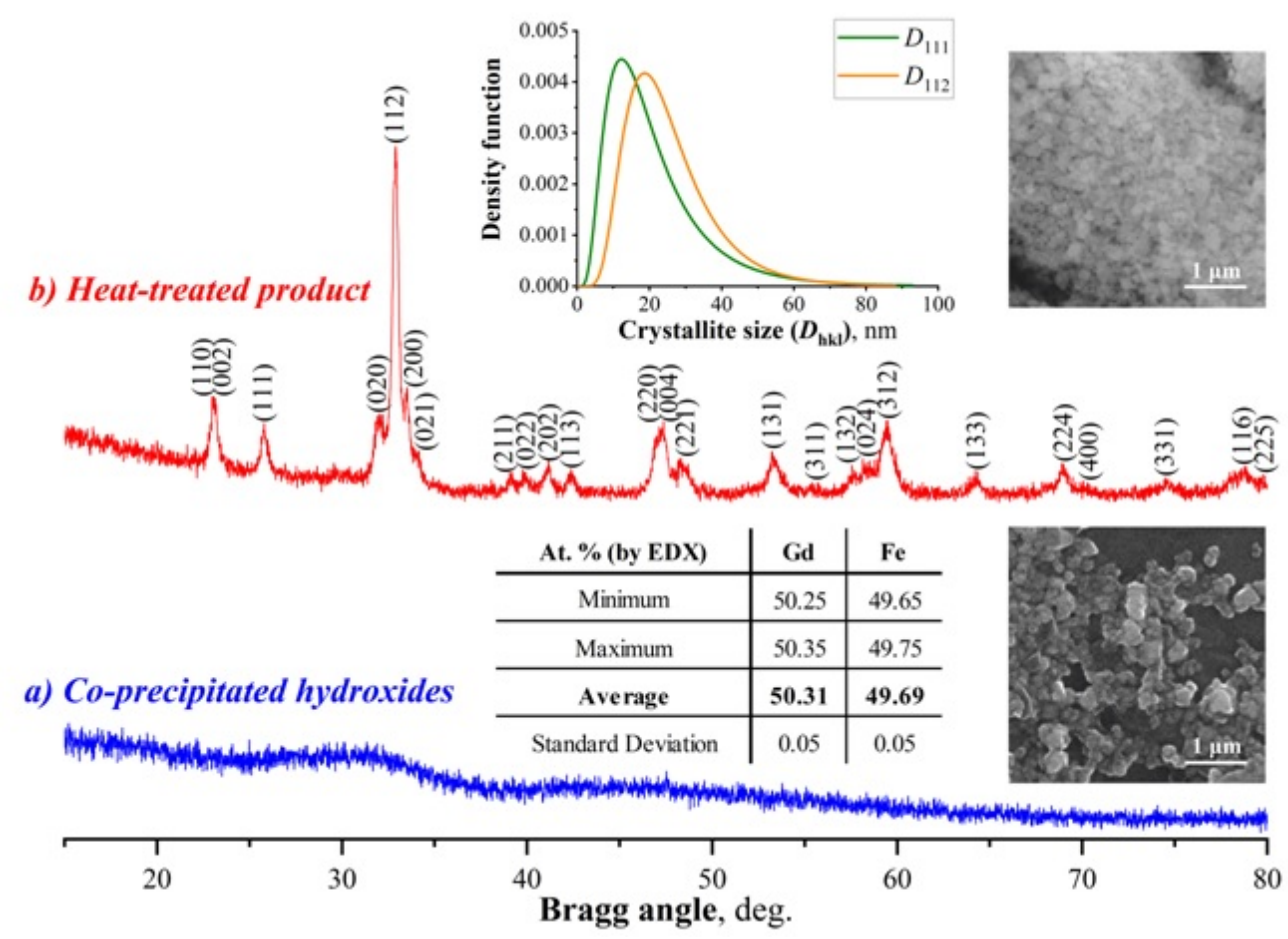

FIG. 2. The PXRD and SEM results of the obtained co-precipitated hydroxides $(a)$ and heat-treated product $(b)$. The insert in $(a)$ shows the EDX results of the co-precipitated hydroxides, and the insert in $(b)$ shows the lognormal size distribution of the prepared $\mathrm{GdFeO}_{3}$ nanocrystals

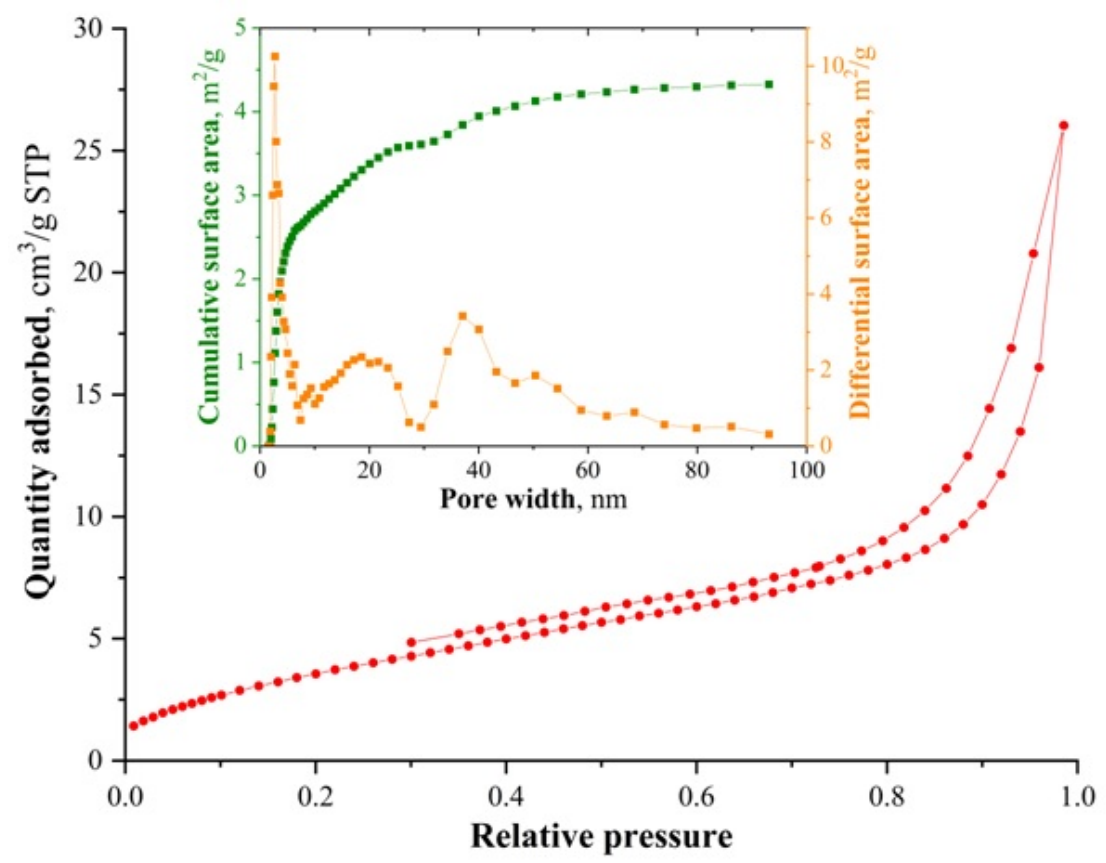

FIG. 3. The nitrogen adsorption-desorption isotherm of the heat-treated product. The insert shows the pore width distribution in the heat-treated product 
of pores of different sizes between individual nanocrystals in their agglomerates, which is consistent well with the results of SEM analysis. Based on the adsorption-desorption data obtained, it was found that the BET surface area is about $13.4 \mathrm{~m}^{2} / \mathrm{g}$, the total pore volume is about $40.3 \mathrm{~mm}^{3} / \mathrm{g}$, and the average pore width is approximately $12 \mathrm{~nm}$. The cumulative and differential surface areas are presented as an insert in Fig. 3. The appearance of mesopores and macropores in the analyzed sample is due to the spacings formed by the agglomerates of $\mathrm{GdFeO}_{3}$ nanoparticles. The characteristic size of the nanoparticles obtained based on the specific surface area $\left(13.4 \mathrm{~m}^{2} / \mathrm{g}\right)$ and X-ray density $\left(7.30 \mathrm{~g} / \mathrm{cm}^{3}\right)$ of gadolinium orthoferrite, was found to be about $62 \mathrm{~nm}$. This value is significantly larger than the average crystallite size $(23 \pm 3 \mathrm{~nm})$ and almost equal to the maximum crystallite size in the lognormal size distribution presented above $(\sim 65-70 \mathrm{~nm}$ ) (see insert in Fig. $2 b$ ) which indicates a strong agglomeration of nanocrystals with grain boundaries inaccessible to nitrogen.

For a better understanding of the structural and morphological features of $\mathrm{GdFeO}_{3}$ nanocrystal agglomerates in the synthesized sample, the size distribution of particles in the prepared colloidal solution was analyzed by DLS and it is presented in Fig. 4.

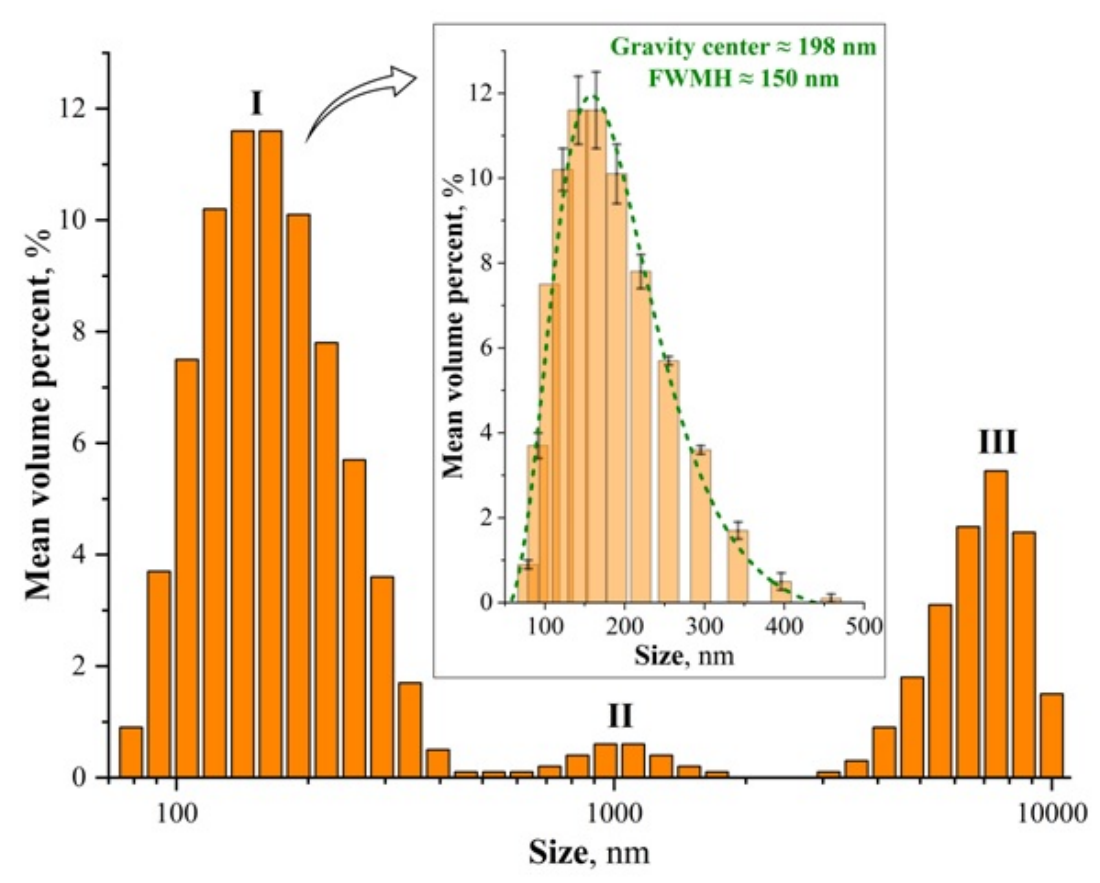

FIG. 4. The size distribution of particles in the prepared colloidal solution of the heat-treated product as shown by DLS

As one can see, the obtained distribution is trimodal, so the particles in the analyzed colloidal solution can be assigned to three peaks: I, II and III, which correspond to primary, secondary and tertiary agglomerates of $\mathrm{GdFeO}_{3}$ nanocrystals, respectively. Approximately $75 \%$ of the particles belong to the primary agglomerates (peak I, which has a gravity center of about $198 \mathrm{~nm}$ and a full width at half maximum (FWHM) of about $150 \mathrm{~nm}$ ). The remaining particles have larger sizes and belong to the higher-order agglomerates. These results indicate that the synthesized $\mathrm{GdFeO}_{3}$ nanoparticles tend to agglomerate in the prepared colloidal solution, and the resulting agglomerates, in turn, tend to agglomerate with each other to form larger agglomerates. In other words, the particles that belong to the peak I are agglomerates of the nanoparticles, the particles that belong to the peak II are agglomerates of the particles of peak I, and the particles that belong to the peak III are agglomerates of the particles of peak II. It should be noted that according to the presented results, particles with nanocrystal sizes were not found at all in the prepared colloidal solution, despite the lengthy procedure of high-energy ultrasonic dispergation. This fact indicates that in this sample gadolinium orthoferrite is present in the form of stable clusters of about $198 \mathrm{~nm}$ in size of $\mathrm{GdFeO}_{3}$ nanocrystals of about $23 \mathrm{~nm}$. Thus, according to a rough estimate, each such cluster contains about 640 individual nanocrystals. It is worth to be noted that a part of the nanocrystals in these clusters is more tightly coupled, as evidenced by the lower characteristic particle size $(\sim 62 \mathrm{~nm})$ estimated from the specific surface area of the sample. The tendency of nanocrystals to form clusters and their high stability can be partially explained by their small absolute value of zeta potential which was found to be equal to $1 \mathrm{mV}$. 
To determine the features of the magnetic structure of the synthesized gadolinium orthoferrite nanocrystals the Mössbauer spectroscopy of the heat-treated product was performed and it is presented in Fig. 5.

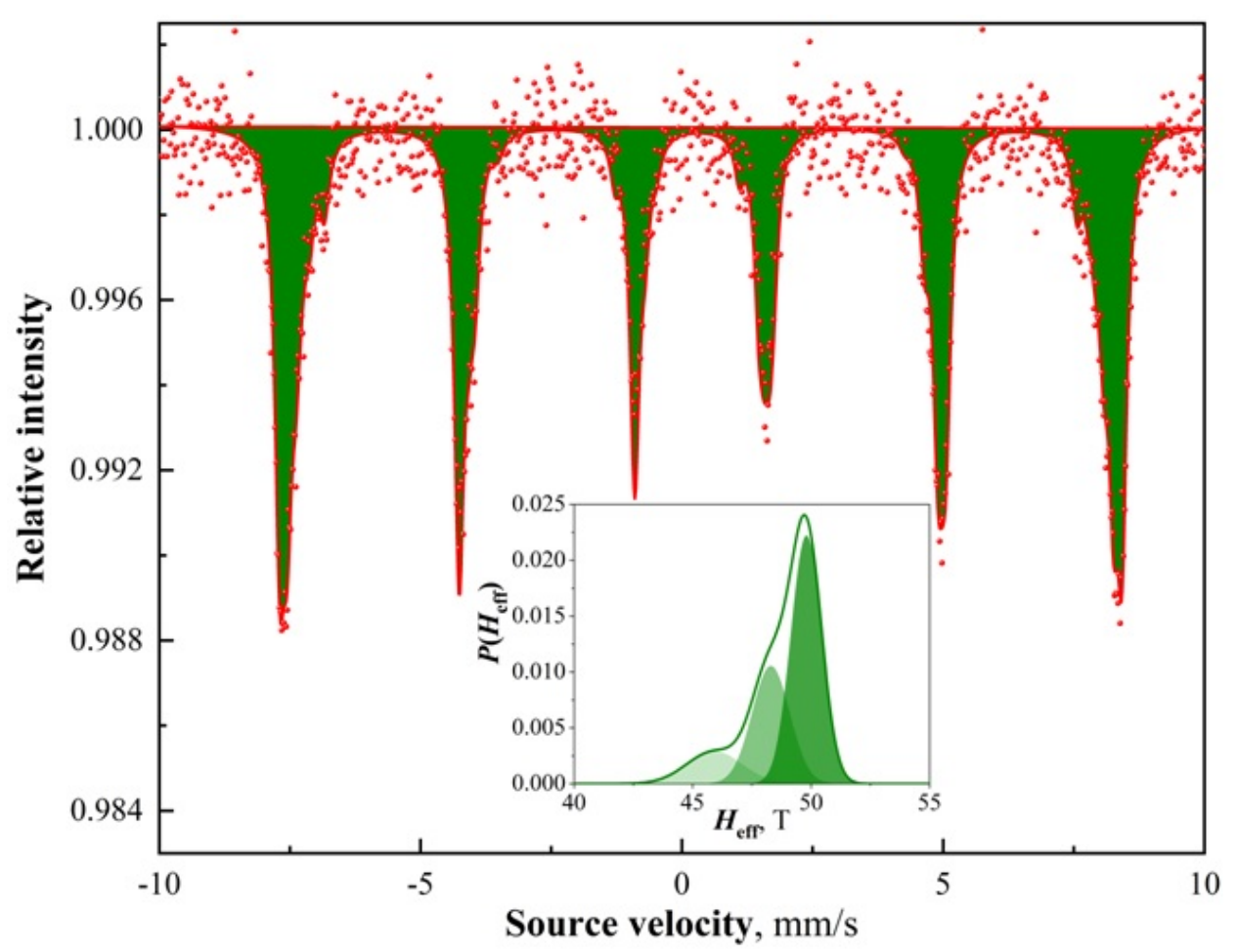

FIG. 5. The results of Mössbauer spectroscopy of the heat-treated product. The insert shows the magnetic hyperfine field distribution

According to the presented results, the heat-treated product contains magnetic (sextet) component. The hyperfine field of the total sextet component corresponds with those of orthorhombic gadolinium orthoferrite [23]. The magnetically ordered phase of $o-\mathrm{GdFeO}_{3}$ has a trimodal distribution, as follows from the results presented as an insert in Fig. 5. As it is well-known, gadolinium orthoferrite belongs to the typical antiferromagnets with weak ferromagnetism resulting from a slight distortion of the crystal structure of $\mathrm{GdFeO}_{3}$ from the perovskite structure with the corresponding canting of the $\mathrm{Fe}^{3+}$ spins from antiparallelism. Thus, we can assume the presence of two magnetically ordered iron sublattices with close effective magnetic field values, which are the components $\mathrm{S} 1(49.8 \pm 0.1 \mathrm{~T})$ and $\mathrm{S} 2(48.3 \pm 0.1 \mathrm{~T})$ with almost equal amount proportions. But additionally, it is also observed the presence of a third sextet component S3 with a noticeably lower effective magnetic field $(45.9 \pm 0.1 \mathrm{~T})$, which may correspond to the contribution of the distorted crystal and magnetic structure of the nanoparticle surface. Thus, all of the sextet components belong to the $\mathrm{GdFeO}_{3}$ magnetically ordered phase and related to complex structural and morphological features of synthesized nanocrystals. Detailed characteristics of all the sextet components are summarized in Table 1.

TABLE 1. ${ }^{57} \mathrm{Fe}$ Mössbauer parameters of all the sextet components

\begin{tabular}{|c|c|c|c|c|}
\hline $\begin{array}{c}\text { Sextet } \\
\text { component }\end{array}$ & $\begin{array}{c}\text { Isomer shift, } \\
\mathrm{mm} / \mathrm{s}\end{array}$ & $\begin{array}{c}\text { Quadrupole } \\
\text { splitting, mm/s }\end{array}$ & $\begin{array}{c}\text { Effective } \\
\text { magnetic field, T }\end{array}$ & $\begin{array}{c}\text { Total } \\
\text { amount, \% }\end{array}$ \\
\hline$S 1$ & $0.363 \pm 0.002$ & $0.008 \pm 0.003$ & $49.8 \pm 0.1$ & 55.1 \\
\hline$S 2$ & $0.355 \pm 0.005$ & $0.011 \pm 0.003$ & $48.3 \pm 0.1$ & 32.0 \\
\hline$S 3$ & $0.351 \pm 0.007$ & $0.038 \pm 0.011$ & $45.9 \pm 0.1$ & 12.9 \\
\hline
\end{tabular}

The magnetic behavior of the $\mathrm{GdFeO}_{3}$ nanoparticles was investigated at room temperature $(298 \mathrm{~K})$ using vibration magnetometry, the results of which are shown in Fig. 6.

The obtained dependence of magnetization on the applied magnetic field has an S-shaped form with an absence of complete saturation even at high values of the applied magnetic field, which in conjunction with low remanent magnetization $(0.37 \mathrm{emu} / \mathrm{g})$ is a characteristic feature of superparamagnetic nanoparticles [24]. However, high coercivity 


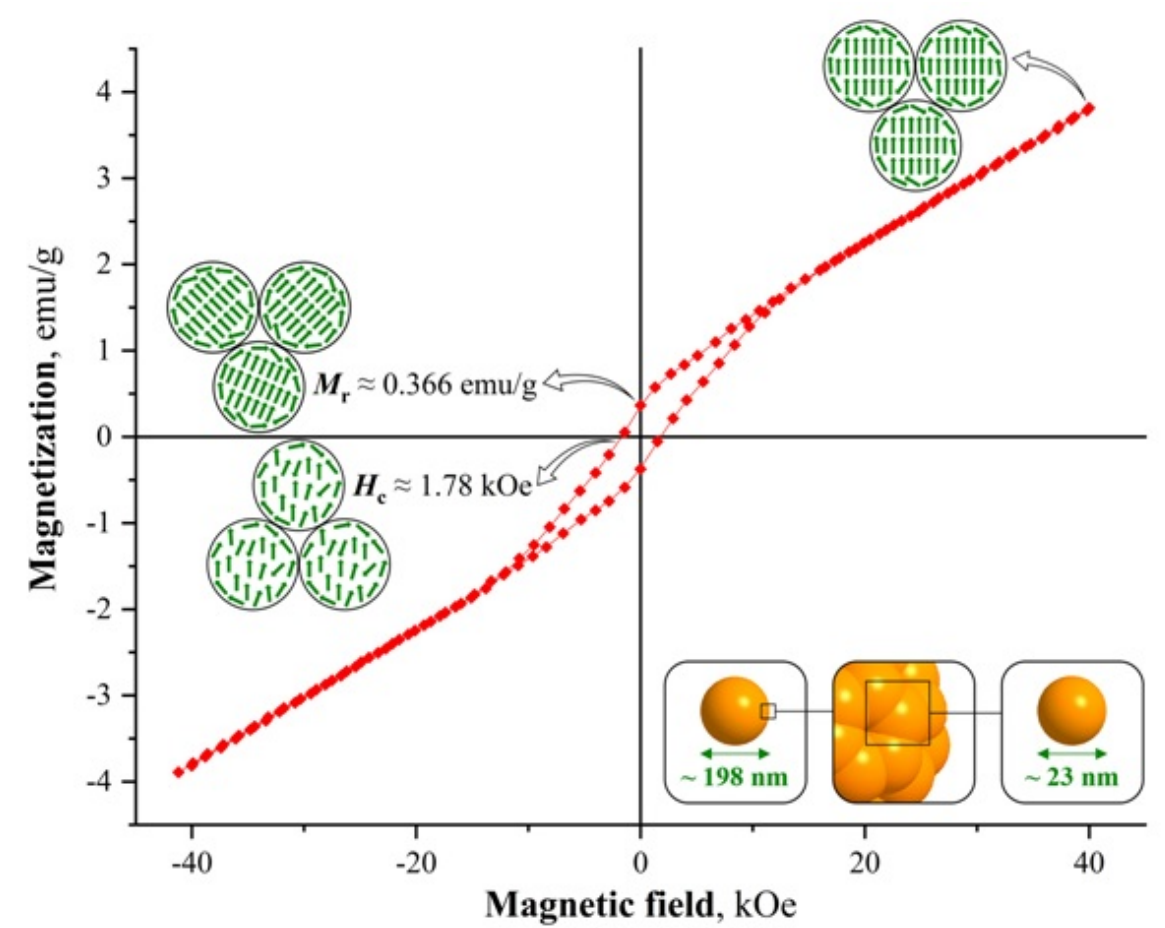

FIG. 6. The magnetization curve of the heat-treated product at $298 \mathrm{~K}$

$(1.78 \mathrm{kOe})$, relatively low magnetization $(3.82 \mathrm{emu} / \mathrm{g})$ at the $40 \mathrm{kOe}$ applied field, and an obvious magnetic hysteresis loop indicate a more complex magnetic structure of this substance. The above information on the strong agglomeration of gadolinium orthoferrite nanocrystals allows us to assume that the observed magnetization dependence is the result of the superparamagnetic nanoparticle cluster's behavior but not individual nanoparticles of $\mathrm{GdFeO}_{3}$. In this case, the resulting behavior of the substance in a changing external magnetic field has the characteristic features of both superparamagnetic and ferromagnetic components. When an external magnetic field about $40 \mathrm{kOe}$ (see insert in Fig. 6) is applied to the clusters of superparamagnetic nanoparticles, their inner magnetic moments tend to align along the applied field, leading to a net magnetization, but magnetic saturation does not occur due to disordered surfaceassociated magnetic moments. When the external magnetic field decreases to zero, a slight residual magnetization $(0.37 \mathrm{emu} / \mathrm{g})$ remains, mainly due to the cluster magnetic moment of strongly coupled $\mathrm{GdFeO}_{3}$ nanoparticles that all together behave like a ferromagnet. Then we must apply a significant demagnetizing field (1.78 kOe) to reduce the magnetization of the sample to zero. A schematic representation of the processes occurring with clusters of superparamagnetic nanoparticles under the external magnetic fields as well as a schematic structure of these clusters are presented as inserts in Fig. 6. As one can see, the behavior of clusters of superparamagnetic nanoparticles is markedly different from the magnetic behavior of a collective of independent superparamagnetic nanoparticles [25].

\section{Conclusion}

Superparamagnetic $\mathrm{GdFeO}_{3}$ nanocrystals with an isometric morphology and an average size of $23 \pm 3 \mathrm{~nm}$ were successfully synthesized by the low-temperature reverse co-precipitation of gadolinium and iron(III) hydroxides followed by their heat treatment in the air. Analysis results indicate a strong agglomeration of gadolinium orthoferrite nanocrystals in the form of stable clusters with a size of about $200 \mathrm{~nm}$ on average. This cluster structure of $\mathrm{GdFeO}_{3}$ possesses its weak ferromagnetic behavior in contrast to the superparamagnetic behavior of individual nanocrystals. Besides, a colloidal solution, produced by water-assisted sonicating of gadolinium orthoferrite nanopowder, contains the first-, second-, and third-order agglomerates of the initial $\mathrm{GdFeO}_{3}$ nanocrystals, and thus a novel approach for colloidal solution preparation should be proposed for further MRI contrast applications.

\section{Acknowledgments}

The research was supported by the Russian Science Foundation (project No. 19-73-00286). The study was partially performed on the equipment of the Engineering Center of Saint Petersburg State Institute of Technology. The 
authors are grateful to Rogozev A. B. for the ${ }^{57} \mathrm{Fe}$ Mössbauer spectroscopy study performed using the equipment of the RITVERC JSC company.

\section{References}

[1] Geraldes C.F.G.C., Laurent S. Classification and basic properties of contrast agents for magnetic resonance imaging. Contrast Media \& Molecular Imaging, 2009, 4(1), P. 1-23.

[2] Xiao Y.-D. et al. MRI contrast agents: Classification and application (Review). International Journal of Molecular Medicine, 2016, 38(5), P. 1319-1326.

[3] Estelrich J., Sánchez-Martín M.J., Busquets M.A. Nanoparticles in magnetic resonance imaging: From simple to dual contrast agents. International Journal of Nanomedicine, 2015, 10, P. 1727-1741.

[4] Szpak A. et al. $\mathrm{T}_{1}-\mathrm{T}_{2}$ Dual-modal MRI contrast agents based on superparamagnetic iron oxide nanoparticles with surface attached gadolinium complexes. Journal of Nanoparticle Research, 2014, 16(11), P. 2678.

[5] De M. et al. Hybrid magnetic nanostructures (MNS) for magnetic resonance imaging applications. Advanced Drug Delivery Reviews, 2011, 63(14-15), P. 1282-1299.

[6] Pinho S.L.C. et al. Synthesis and Characterization of Rare-Earth Orthoferrite $\mathrm{LnFeO}_{3}$ Nanoparticles for Bioimaging. European Journal of Inorganic Chemistry, 2018, 2018(31), P. 3570-3578.

[7] Söderlind F. et al. Colloidal synthesis and characterization of ultrasmall perovskite $\mathrm{GdFeO}_{3}$ nanocrystals. Nanotechnology, 2008, 19(8), P. 085608.

[8] Athar T. et al. Super paramagnetic iron oxide and gadolinium $\left(\mathrm{FeGdO}_{3}\right)$ nanopowder synthesized by hydrolytic approach passes high level of biocompatibility and MRI-based dual contrast property for competent molecular imaging and therapeutic interventions. Biomedical Physics \& Engineering Express, 2016, 2(2), P. 025010.

[9] Deka S. et al. Synthesis, characterization and in vitro analysis of $\alpha-\mathrm{Fe}_{2} \mathrm{O}_{3}-\mathrm{GdFeO}_{3}$ biphasic materials as therapeutic agent for magnetic hyperthermia applications. Materials Science and Engineering: C, 2018, 92, P. 932-941.

[10] Caravan P. et al. Gadolinium(III) Chelates as MRI Contrast Agents: Structure, Dynamics, and Applications. Chemical Reviews, 1999, 99(9), P. 2293-2352.

[11] Hermann P. et al. Gadolinium(III) complexes as MRI contrast agents: ligand design and properties of the complexes. Dalton Transactions, 2008, 23, P. 3027-3047.

[12] Zhao X. et al. Ultrasmall Superparamagnetic Iron Oxide Nanoparticles for Magnetic Resonance Imaging Contrast Agent. Journal of Nanoscience and Nanotechnology, 2014, 14(1), P. 210-220.

[13] Dulicska-Litewka J. et al. Superparamagnetic Iron Oxide Nanoparticles-Current and Prospective Medical Applications. Materials (Basel), 2019, 12(4), P. 617.

[14] Popkov V.I. et al. The Formation of Nanocrystalline Orthoferrites of Rare-Earth Elements $\mathrm{XFeO}_{3}(\mathrm{X}=\mathrm{Y}, \mathrm{La}$, Gd) Via Heat Treatment of Coprecipitated Hydroxides. Russian Journal of General Chemistry, 2017, 87(11), P. 2516-2524.

[15] Tugova E.A., Zvereva I.A. Formation mechanism of $\mathrm{GdFeO}_{3}$ nanoparticles under the hydrothermal conditions. Nanosystems: physics, chemistry, mathematics, 2013, 4(6), P. 851-856.

[16] Gimaztdinova M.M. et al. Glycine-nitrate combustion synthesis of $\mathrm{GdFeO}_{3}$ nanocrystals. Condensed Phases and Interfaces, 2016, 18(3), P. 422-431.

[17] Li L. et al. Synthesis, Photocatalytic and Electrocatalytic Activities of Wormlike GdFeO 3 Nanoparticles by a Glycol-Assisted Sol-Gel Process. Industrial \& Engineering Chemistry Research, 2013, 52(26), P. 9130-9136.

[18] Tang P. et al. Preparation of Nanocrystalline $\mathrm{GdFeO}_{3}$ by Microwave Method and Its Visible-light Photocatalytic Activity. Integrated Ferroelectrics, 2014, 153(1), P. 73-78.

[19] Tugova E.A., Karpov O.N. Nanocrystalline perovskite-like oxides formation in $\mathrm{Ln}_{2} \mathrm{O}_{3}-\mathrm{Fe}_{2} \mathrm{O}_{3}-\mathrm{H}_{2} \mathrm{O}$ ( $\mathrm{Ln}=\mathrm{La}$, Gd) systems. Nanosystems: physics, chemistry, mathematics, 2014, 5(6), P. 854-860.

[20] Mathur S. et al. Nanocrystalline Orthoferrite $\mathrm{GdFeO}_{3}$ from a Novel Heterobimetallic Precursor. Advanced Materials, 2002, 14(19), P. 14051409.

[21] Popkov V.I., Almjasheva O. V. Formation mechanism of $\mathrm{YFeO}_{3}$ nanoparticles under the hydrothermal conditions. Nanosystems: physics, chemistry, mathematics, 2014, 5(5), P. 703-708.

[22] Popkov V.I. et al. Formation Mechanism of Nanocrystalline Yttrium Orthoferrite under Heat Treatment of the Coprecipitated Hydroxides. Russian Journal of General Chemistry, 2015, 85(6), P. 1370-1375.

[23] Romero M. et al. Synthesis by molten salt method of the $\mathrm{AFeO}_{3}$ system $(\mathrm{A}=\mathrm{La}, \mathrm{Gd})$ and its structural, vibrational and internal hyperfine magnetic field characterization. Physica B: Condensed Matter, 2014, 443, P. 90-94.

[24] Popkov V.I., Tolstoy V.P., Semenov V.G. Synthesis of phase-pure superparamagnetic nanoparticles of $\mathrm{ZnFe}_{2} \mathrm{O}_{4}$ via thermal decomposition of zinc-iron layered double hydroxysulphate. Journal of Alloys and Compounds, 2020, 813, P. 152179.

[25] Albadi Y., Popkov V.I. Dual-modal contrast agent for magnetic resonance imaging based on gadolinium orthoferrite nanoparticles: synthesis, structure and application prospects. Medicine: Theory and Practice, 2019, 4(S), P. 35-36. 